\title{
Application of impedance method for research the dynamics of asynchronous motors
}

\author{
Boris D. Khastsaev*, and Dmitry $V$. Merkushev \\ North-Caucasus mining and metallurgical Institute (state technological University), Vladikavkaz, \\ Russia
}

\begin{abstract}
One of the possible fields of application of the impedance method based on the use of impedance and its components as an informative parameter is considered. This area of work is the study and diagnosis of asynchronous motors in dynamic mode. The paper presents both a $\mathrm{T}$ - shaped scheme of replacement of an asynchronous motor and one of its possible mathematical models, on the basis of which conclusions were made about the feasibility of applying the impedance method for the study and diagnosis of asynchronous motors and the need to develop hardware for this method. In this regard, the main part of the work is devoted to the consideration of measuring transducers necessary for both the technical implementation of the method as a whole, and the instrument support of the method. The option to build transmitters that use the basis four-armmeasuring bridge circuit known as a Wheatstone bridge. The first converter is easier to implement because it is implemented on the basis of the Whitstonbridge with one feedback, but is characterized by less functionality. The second converter is implemented more difficult, as it is also implemented on the basis of the Whitstonbridge, but with two feedbacks. However, this version of the converter has more functionality.
\end{abstract}

\section{Introduction}

There is a wide application of the impedance method in medicine and biology for scientific and diagnostic studies. At the same time, the effectiveness of these studies largely depends on the technical support of this method, since the method provides the formation of primary information related to the properties of the studied and diagnosed objects and the changes occurring in them [1-4]. A similar problem is set and solved in the work, but for the study of the dynamics of technical objects. At the same time, an asynchronous motor (AM) is considered as an object of research and diagnosis, the high value of which in the national economy is not in doubt.

The application of the impedance method for the study and diagnosis of AM is an important problem, since the practical application of the method involves providing with high reliability the identification of a number of am defects. It is very economically profitable.

Thus, the work devoted to the use of the impedance method for the study and diagnosis of AM, the development of means of technical support of this method is actual work.

\footnotetext{
* Corresponding author: bordsamchas@rambler.ru
} 


\section{Theoretical part}

It is known that in its performance AM has great advantages over other types of electrical machines, as it is characterized by high reliability, the absence of any switching elements, a large interval of time between preventive maintenance. However, AM is the most complex engine and is a complex nonlinear control object. But with the advent of microprocessor technology, electric drives based on AM successfully displace direct currentelectric drives. This shows the importance and necessity of research and diagnosis of hypertension, the study of dynamic characteristics with high reliability, for the implementation of which there is currently no technical provision with high user characteristics. Therefore, the purpose of this work is to fill the gap and the development of technical means for the study of the dynamics of AM with improved characteristics.

The analysis of literary sources showed the necessity of creating a mathematical model of AM and examining the equivalent circuits of the AM. It also showed the existence of a large number of both models and the substitution scheme, so the authors chose the most popular replacement scheme of AM, shown in fig.1.

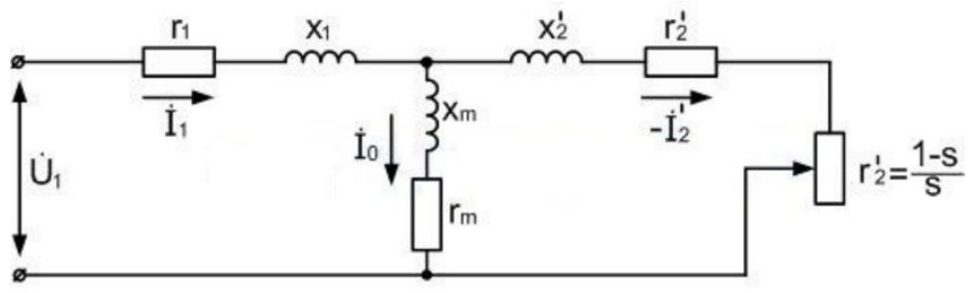

$\mathrm{T}$ - shaped scheme of replacement

Fig. 1. The variant of the equivalent circuit AM.

For fig. 1 applied the following notation: $U_{1}-$ the voltage vector of the stator; $I_{1}-$ the vector of stator current; $I_{0}$ - is the vector of the magnetizing current; $I_{2}^{1}{ }_{2}$ - is the vector of the rotor current; $r_{1}, r^{1}{ }_{2}$ - active resistance of stator and rotor; $x_{1}, x_{2}^{1}{ }_{2}$ - inductance dissipation of the stator and rotor; $x_{m}$ is the mutual inductance; $\mathrm{s}$ is the slip of the motor.

Now we present one of the variants of mathematical models of AM.

The system of equations describing AM with a short-circuited rotor in a fixed system, given in [5], has the form:

$$
\left\{\begin{array}{l}
\bar{u}_{s}=r_{s} \cdot \bar{i}_{s}+\frac{1}{\Omega_{\delta}} \cdot \frac{d \bar{\Psi}_{s}}{d t} ; \\
0=r_{r} \cdot \bar{i}_{r}+\frac{1}{\Omega_{\delta}} \cdot \frac{d \bar{\Psi}_{r}}{d t}-j \omega \cdot \bar{\Psi}_{r} ; \\
\bar{\Psi}_{s}=\left(l_{m}+l_{s \sigma}\right) \cdot \bar{i}_{s}+l_{m} \cdot \bar{i}_{r} ; \\
\bar{\Psi}_{r}=\left(l_{m}+l_{r \sigma}\right) \cdot \bar{i}_{r}+l_{m} \cdot \bar{i}_{s} ; \\
m=\zeta_{N} \cdot k_{r} \cdot\left(\Psi_{r a} \cdot i_{s \beta}-\Psi_{r \beta} \cdot i_{s a}\right) ; \\
m-m_{\alpha}=T_{j} \frac{d \omega}{d t} .
\end{array}\right.
$$

Without stopping at a detailed analysis of the equivalent circuit and a detailed description of the above system of equations, we note that all physical quantities contained in both the equivalent circuit and in the system of equations that provide the study of AM dynamics can be measured and controlled by measuring converters of impedance parameters (IPC). 
It is possible to use sensors with their connection to the input of these converters. Thus, the development of IPC for the study of am dynamics is an important task and the development of IPC with improved characteristics will serve as the basis for the widespread use of the impedance method for the study of AM dynamics. The relevance and importance of a highly effective method for the study and diagnosis of AM to the present time remain at a high level, which is confirmed by scientific publications [6-10]. This determined both the relevance and the purpose of the work devoted to the promotion of the impedance method for the study of AM dynamics and the issues of IPC construction necessary for the practical application of the method in question.

\section{Converter for measuring inductance and active resistance}

The IPC scheme is shown in fig.2, from which it can be seen that IPC is built on a four-arm bridge measuring circuit (BC) with the supply voltage of the unbalance and the supply voltage of the $\mathrm{BC}$ power supply to the $\mathrm{BC}$ branch with an adjustable element [6]. This is the upper branch of $\mathrm{BC}$, and the lower branch of $\mathrm{BC}$ contains an object with the parameters $\mathrm{L}_{\mathrm{X}}$ and $\mathrm{R}_{\mathrm{X}}$.

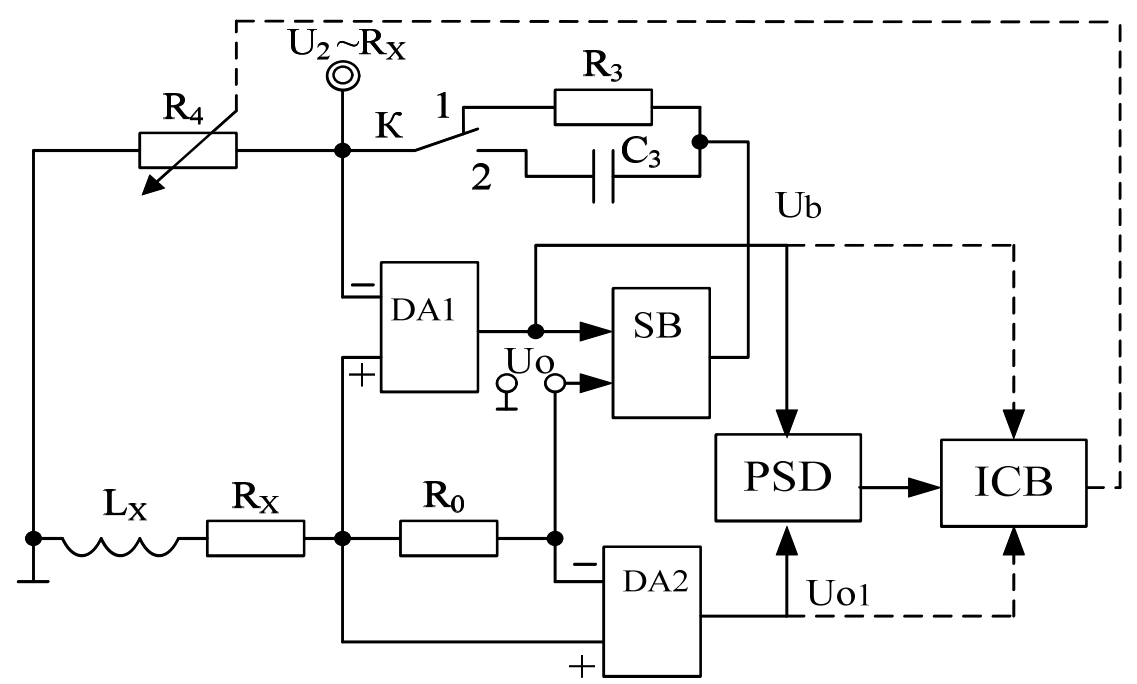

Fig. 2. Scheme IPC to measure DKH and KKH with intelligent control block (ICB).

Used in the scheme designations: $L_{x}$ and $R_{x}$ - respectively, the inductance and resistance of the object; $R_{O}$ - resistance of the sample element; $R_{3}$ - resistance of the shoulder of the comparison branch resistor, which provides the choice of the value of the conversion $R_{x}$; $C_{3}$ - capacitance of the comparison branch capacitor, which provides the choice of the converted value $R_{x} ; R_{4}$ - the resistance of the adjustable resistor of the comparison branch, which ensures the achievement of the quasi-equilibrium state; DA1-differential amplifier, which provides the formation of the unbalance signal BC-Ub; DA2 is a differential amplifier, forming part of the main voltage $-U_{O I}$; PSD-phase sensitive detector, providing a signal for adjusting the resistance value of adjustable resistor; $U_{O}$ - the reference voltage for the power $\mathrm{BC} ; U_{2}$ - the voltage generated at the shoulder of a branch comparison (the voltage drop across adjustable resistor) at the time of quasireverse and proportionally one of the converted values of $L_{x}$ and $R_{x} ; \mathrm{K}-$ key.

In addition, we note that the controlled (adjustable) resistor $R_{4}$ serves as an adjustable element. If the key $\mathrm{K}$ is in position 2, then the inductance is converted, and if in position 1, 
then the active resistance $R_{x}$. In the latter case, when applying to the input of the adder $\mathrm{SB}$, the constant voltage on the isarm is formed by an analog value proportional toresistance $R_{x}$. The reference voltage is the voltage drop on the resistance Ro. The expression for the output voltage $U b$ IPC in general can be represented as:

$$
U b=U o \frac{Z_{x} G_{0}-R_{4} G_{3}}{1+Z_{X} G_{0}}
$$

The expression for the output voltage $U b$ in the position of the key 2 will have the form:

$$
U_{b}=U o \frac{\left(j \omega L_{X}+R_{X}\right) G_{0}-R_{4} j \omega C_{X}}{1+\left(j \omega L_{X}+R_{X}\right) G_{0}}
$$

and the expression for the same voltage in the position of the key 1 will have the form:

$$
U_{b}=U_{O} \frac{\left(j \omega L_{X}+R_{X}\right) G_{0}-R_{4} G_{3}}{1+\left(j \omega L_{X}+R_{X}\right) G_{0}}
$$

From the last two expressions it can be seen that when the inductance of the object is converted, its value is determined from the following quasi-equilibrium condition:

$$
R_{4}=L_{x} G_{o} / C_{3}
$$

and the analog value $U_{2}$ equal to the expression:

$$
U_{2}=U_{O} L_{x} G_{o} / C_{3}
$$

will be proportional to $L_{x}$.

In the conversion of the resistance $R_{X}$, the value of the resistance is determined from the following quasi-equilibrium condition:

$$
R_{4}=R_{x} G_{o} / R_{3}
$$

the analog value $U_{2}$ in this case is equal to the expression:

$$
U_{2}=R_{x} U_{\ni} G_{o} / R_{3}
$$

will be proportional to $R_{x}$.

IPC capabilities can be significantly improved if it is supplemented with a block of intelligent control (ICB), designed to solve a number of problems arising in the study of objects not previously studied and electrical circuits which have not yet been known. Such a block is also important for the processing of research results, their accumulation and effective use.

\section{IPC for multi-point measurement of AM}

Multipoint measurement of impedance parameters involves the use of a large number of electrodes connected to a single measuring circuit, as in IPC used BC with a linearized output characteristic [10]. Another important property of the measuring circuit used is its use in the quasi-equilibrium mode to ensure high accuracy of measurement of the main component of the impedance and lower accuracy of the secondary.

A structure schemeof IPC, designed to measure two impedance parameters at many points of the object, is shown in fig.3, which shows that the number of electrodes is $n$. The electrodes should be located at some distance from each other. IPC also contains the shaper of the rectangular impulses (RIS), a binary pulse counter (BC), BC built using two 
operational amplifiers (OA1 and OA2), the source of measuring signals (SMS), a phasesensitive detector (PSD), block of regulating(BR), a block of intellectual processing of the measured data (IB), the module information display (IDM) and two multiplexers (Ms1) and (Ms2) informational (I) and the address inputs (A).

The lower branch consists of the shoulder with the impedances $Z_{3}$ and $Z_{4}$, and the top of the shoulder with the impedance $Z_{l}$ and of the shoulder with the impedance plots of the investigated object from $Z_{x l}$ to $Z_{x(n-1)}$.

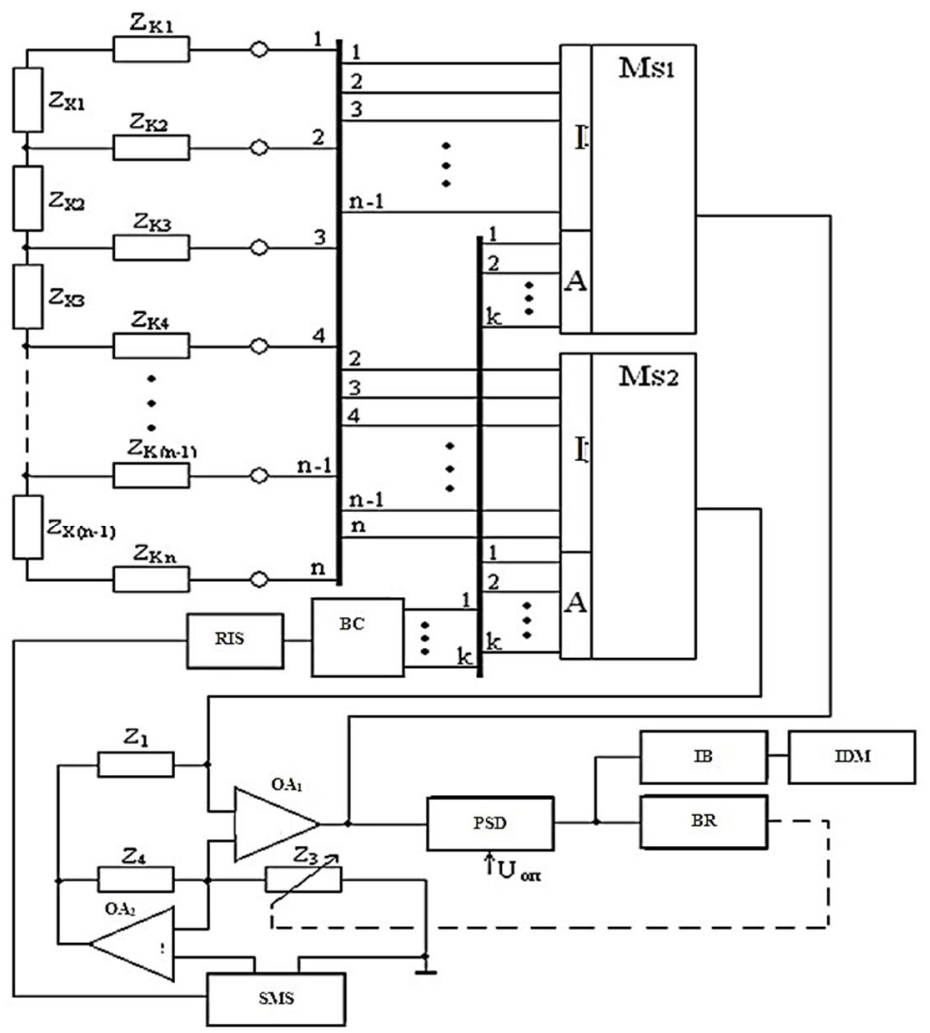

Fig. 3. IPC for multi-point measurements of parameters of AM.

SMS provides $\mathrm{BC}$ with different shapes of measurement signals and different frequencies, resulting in enhanced IPC capabilities and applications. For linearization of the output characteristic BC, operational amplifiers with negative feedback circuits are used. The output voltage of the used $\mathrm{BC}$ corresponds to the expression:

$$
U_{b}=U_{o}\left(Y_{1} Z_{x i}-Y_{4} Z_{3}\right),
$$

where: $U b$ is the output voltage OA2, $U o$ is the output voltage SMS, $Y_{1}$ and $Y_{4}$ - shoulder 1 and 4 admittance $\left(Y_{1}=1 / Z_{1}\right.$ and $\left.Y_{4}=1 / Z_{4}\right), Z_{3}$ is the impedance of the 3-rd arm, adjustable BR.

The impedance between two electrodes, i.e. the measured value is most often: $\left(\operatorname{Re} Z_{x i}+\right.$ $\left.j \operatorname{Im} Z_{x i}\right)$. For these cases, the output voltage OA2, respectively, can be represented as: $U_{b}=U_{o}\left[Y_{1}\left(\operatorname{Re} Z_{x i}+j \operatorname{Im} Z_{x i}\right)-Y_{4} Z_{3}\right]$. 
From the expression it is seen that the impedance $Z_{3}$ should be an active resistance and its regulation achieves the state of quasi-reverse $\mathrm{BC}$, and thus receive par $-Y_{I} \operatorname{Re} Z_{x i}=Y_{4} \operatorname{Re} Z_{3}$, which is easily determined value $R e Z_{x i}$.

Simultaneously, the voltage at the output OA2 becomes directly proportional to the reactive component of the measured impedance, i.e $U_{b g}=j U_{o} Y_{I} \operatorname{Im} Z_{x i}$.

IB in IPC is designed to determine other informative parameters of the impedance, and IDM provides a visual display of the controlled information. Switching of information inputs $\mathrm{Ms}_{1}$ and $\mathrm{Ms}_{2}$ is provided by signals from $\mathrm{BC}$, which receives pulses from RIS. In turn, RIS receives signals from SMS, from which RIS provides the formation of short rectangular pulses. One of the important properties of IPC is also the ability to eliminate the impact on the measurement accuracy of a number of uninformative parameters shown in general in the figure as $Z_{k i \ldots} Z_{k n}$. These parameters can include impedance of connecting wires, impedance of contacts and electrodes, as well as impedance of boundary layers, etc.

\section{Conclusion}

The paper notes the importance of using the impedance method for the study and diagnosis of objects. In this regard, the aim of the study of the capabilities of the impedance method for the study and certification of the dynamic characteristics of $\mathrm{AD}$ and the results were obtained, which are of significant practical importance. To a certain extent, the work contributes to the promotion of an important method of research and diagnosis in the field of electrical machines. Hence, the expediency of developing a technique for conducting studies of the dynamic characteristics of $\mathrm{AD}$, which will be implemented in future works of the authors. It is important to note that it is essential to create a bank of mathematical models and equivalent circuits of $\mathrm{AD}$. This is necessary, first of all, to assess the dynamic characteristics of AD. The paper proposes two options for building IPC to study the dynamics of AD. Developed IPC are characterized by improved properties and capabilities compared to analogues.

The work is of interest to students and graduate students, researchers involved in the development of experimental facilities. The work is of interest to the developers of electronic equipment.

\section{References}

1. B.D. Khastsaev, Kh.Sh. Dryaeva, I.P. Maksimova, M.B. Khastsaev, Devices and systems. Management Control Diagnosis, 2, 25 (2010)

2. B.D. Khastsaev, M.B. Khastsaev, Application information properties of the impedance in medicine and biology, Monograph (LAP LAMBERT Academic Publishing, 2013)

3. B.D. Khastsaev, M.B. Khastsaev, Devices and systems. Management Control Diagnosis, 5, 36 (2010)

4. B.D. Khastsaev, V.A. Sozaev, A.L. Korolev, V.A. Kodzasov, Science and Technology, Scieuro, 222-29 (2016)

5. A.A. Emelyanova et al., Molodoi ushenyi, 13, 20 URL: https://moluch.ru/archive/93/20907/ (2015) (date of circulation: 15.07.2018)

6. B.D. Khastsaev, M.B. Khastsaev, MATERIALS for the IX international scientific practical conference "SCIENTIFIC POTENTIAL FOR LIGHT-2013", 21, 91 (2013)

7. B.D. Khastsaev, L.M. Dedegkaeva, A.L. Korolev, A.I. Abaev, Science and Technology, 2, 14 (2016) 
8. B.D. Khastsaev, V.A. Sozaev, A.L. Korolev, V.A. Kodzasov, Science and Technology, 2, 22 (2016)

9. M.A. Gavriluk, E.P. Sogolovsky, Chetyrechplechie mosty peremennogo toka, (Izd-vo "Vishashkola", L'vov, 1975)

10. B.D. Khastsaev, T.S. Kataev, Patent 2104668 C1 RF, MPK ${ }^{6}$ A 61 B 5/05. Mostovoe ustroistvo dlya mnogotochechnogo opredeleniya impedansnyhk harakteristik bioob 'ektov, №96121545/09, Zayavleno 01.11.1996. 